\title{
Differential effects of heat stress on oxidative status of skeletal muscle with different muscle fibre compositions in broiler chicken
}

KEY WORDS: avian uncoupling protein, superoxide dismutase, antioxidant status, mitochondria, oxidative stress, broiler chickens

Received: 26 June 2018

Revised: 3 December 2018

Accepted: 28 January 2019
${ }^{1}$ Corresponding author: e-mail: kikusato.m@tohoku.ac.jp

\begin{abstract}
Skeletal muscles are composed of two major muscle fibre types, glycolytic and oxidative, which can be differentiated using their mitochondrial content. Mitochondria are a major generator of reactive oxygen species, and muscles have adapted them to possess oxidative resistance to counteract the oxidative damage. The present study aims to clarify the oxidative tolerance of heat stress (HS) in different types of skeletal muscles of broiler chickens. Exposure of 3-week-old broiler chickens to HS conditions $\left(34^{\circ} \mathrm{C}, 12 \mathrm{~h}\right.$ ) resulted in significantly higher lipid peroxidation in Musculus pectoralis (Pec), which consists entirely of glycolytic muscle fibres (type IIB), than in thermoneutral (TN) birds. This increase did not occur in gastrocnemius (Gas) muscle, which has a lower proportion of type IIB fibres $(65-80 \%)$. HS treatment resulted in significantly higher mitochondrial $\mathrm{H}_{2} \mathrm{O}_{2}$ production in Pec muscle but not in Gas muscle. In both muscles, HS treatment did not alter the gene expression levels of cytosolic antioxidative enzymes, superoxide dismutase (SOD) 1, catalase and glutathione peroxidase-4. In Pec muscle, there was no difference in SOD2 mRNA levels between TN and HS birds, while avian uncoupling protein (avUCP) was significantly down-regulated by HS treatment. Conversely, in the Gas muscle of HS birds, SOD2 mRNA level was significantly increased while avUCP mRNA level was unchanged. Based on this evidence, it is suggested that the glycolytic muscle (e.g., Gas muscle) in broiler chickens is more susceptible to HS-induced oxidative disturbance, in which avUCP and SOD2 may be involved.
\end{abstract}

\section{Introduction}

Skeletal muscles are composed of two major muscle fibre types that are referred to as glycolytic (type II) and oxidative (type I) muscle fibres. A major difference between oxidative and glycolytic muscles is their mitochondrial content. Mitochondria function not only as a major energy plant but also as a reactive oxygen species (ROS) generator within most cells. Oxidative muscles are particularly susceptible to ROS attacks due to their high mitochondrial abundance, and therefore they have 
higher activity of antioxidative enzymes such as superoxide dismutase (SOD), catalase (CAT), and glutathione peroxidase (GPx) (Picard et al., 2012). This leads to specific mitochondrial oxidative phenotypes in histochemically and metabolically different muscle fibres.

Heat stress (HS) is an environmental factor that causes oxidative damage to skeletal muscle tissues in animals. Our previous studies have shown that HS induces oxidative damage (Kikusato et al., 2010), involving excess mitochondrial ROS generation due to a suppression of avian uncoupling protein (avUCP) expression (Kikusato and Toyomizu, 2013). These findings were observed in Musculus pectoralis $(\mathrm{Pec})$ muscle, which consists entirely of glycolytic (type IIB) muscle fibres. Glycolytic muscle fibre has a lower mitochondrial content than oxidative muscle (Hakamata et al., 2018). There have been no comparative studies on oxidative disturbance and resistance in skeletal muscles with different muscle fibre composition of HS-exposed birds.

The present study evaluates the effect of HS on oxidative disturbance in two different types of muscles, Pec muscle and gastrocnemius (Gas) muscle that consists of $65 \%$ type IIB muscle fibres (Williams and Dhoot, 1992). In addition, this study investigates the possible involvement of mitochondrial ROS generation and antioxidative enzymes in HS-induced oxidative damage.

\section{Material and methods}

\section{Animals and experimental design}

The Animal Care and Use Committee of the Graduate School of Agricultural Science, Tohoku University (Japan), approved all procedures, and every effort was made to minimize animal pain or discomfort.

Twelve 0-day-old male broiler chickens (Ross strain, Gallus gallus domesticus) were obtained from a commercial hatchery (Matsumoto Poultry Farms and Hatcheries Co., Ltd., Zao, Miyagi, Japan). Animals were housed in electrically-heated batteries under continuous light for 14 days and provided ad libitum access to water and a standard diet for meattype chickens (crude protein 21\%; metabolizable energy $3100 \mathrm{kcal} / \mathrm{kg}$ ), with each ingredient meeting National Research Council (NRC, 2014) recommendation levels. Thereafter, chickens were randomly divided into two groups and moved to individual wire cages. At 25 day of age, the control group was maintained at thermoneutral conditions
(TN) $\left(24^{\circ} \mathrm{C}\right)$ while the other group was exposed to HS conditions $\left(34^{\circ} \mathrm{C}\right)$ for $12 \mathrm{~h}$. Both conditions were kept at $55-65 \%$ relative humidity. After the HS treatment, rectal and muscle temperature were measured by using digital thermometer equipped with a needle type probe. The birds were euthanized by decapitation, and the Pec and Gas muscles were excised and placed in an ice-cold medium comprising $100 \mathrm{mM} \mathrm{KCl}, 50 \mathrm{mM}$ tris(hydroxymethyl) aminomethane (Tris)/ $\mathrm{HCl}(\mathrm{pH} 7.4)$ and $2 \mathrm{mM}$ ethylene glycol-bis( $\beta$-aminoethyl ether)- $N, N, N^{\prime}, N^{\prime}$ tetraacetic acid (EGTA) for mitochondrial isolation (see below). The muscles were frozen, powdered in liquid nitrogen, and stored at $-80^{\circ} \mathrm{C}$ until analysis.

\section{Isolation of skeletal muscle mitochondria}

Skeletal muscle mitochondria were isolated by homogenization, protein digestion and differential centrifugation at $4{ }^{\circ} \mathrm{C}$, as described previously (Kikusato and Toyomizu, 2015b). The protein concentration of the isolated mitochondria was determined using the bicinchoninic acid assay, with bovine serum albumin as the standard. All mitochondria were freshly prepared on the day of the experiment. The respiratory control ratio (RCR) of each mitochondrial sample was measured by using $73 \mu \mathrm{M}$ ADP to examine mitochondrial quality, as previously described (Kikusato and Toyomizu, 2015). The RCR is a ratio of the mitochondrial $\mathrm{O}_{2}$ consumption rate in the presence of ADP to the rate in its absence. The RCR values for succinate-supported respiration were approximately $2.6-2.9$ in all mitochondrial samples, meaning that the isolated mitochondria were of good quality for functional evaluation.

\section{Determination of mitochondrial ROS production}

Mitochondrial ROS generation rates were determined by measuring the hydrogen peroxide $\left(\mathrm{H}_{2} \mathrm{O}_{2}\right)$ generation rate, which was fluorometrically measured by the oxidation of 10-acetyl-3,7-dihydroxyphenoxazine (Amplex ${ }^{\mathrm{TM}}$ Red, Invitrogen, Carlsbad, CA, USA) coupled to the enzymatic reduction by horseradish peroxidase, as described previously (Kikusato and Toyomizu, 2015).

\section{Measurement of lipid peroxidation}

Muscle lipid peroxidation was determined based on the level of thiobarbituric acid (TBA) reactive substances (TBARS). TBARS values were measured as previously described (Kikusato et al., 2016), and were expressed as nanomoles of malondialdehyde (MDA) per equivalent $g$ of wet tissue. 


\section{Quantification of mRNA expression using Real-Time reverse transcription polymerase chain reaction (RT-PCR)}

Isolation of tissue RNA and synthesis of cDNA were conducted as previously described (Kikusato et al., 2016). Real-time RT-PCR analysis was performed to quantify mRNA using a CFX Connect ${ }^{\mathrm{TM}}$ system (Bio-Rad Laboratories, Hercules, CA, USA). mRNA levels of the following ROS regulating proteins and the transcriptional co-factor were quantified: SOD1, SOD2, CAT, GPx4, avUCP and peroxisome proliferator-activated receptor $\gamma$ coactivator $1-\alpha(\mathrm{PGC}-1 \alpha)$. The expression values were normalized to that of 18S-ribosomal RNA (RN18S). Primer sequences of the genes were described in our other papers (Kikusato et al., 2015; 2016).

\section{Statistical analysis}

All data are presented as the mean \pm standard error (SE) of 6 individual samples. Statistical differences between the TN and HS birds in each muscle were identified using a Student's $t$-test. Differences were considered significant for values of $P<0.05$.

\section{Results and discussion}

In broiler chickens exposed to HS treatment TBARS values in $P e c$ muscle were significantly increased, while in Gas muscle there was no increase noted (Figure 1A). These different responses are due to the different effects of HS on the muscles. The differences in muscle temperature could be pos-
Table 1. Gene expression levels of avian uncoupling protein (avUCP), superoxide dismutase (SOD) 2, SOD1, catalase (CAT), glutathione peroxidase (GPx) 4 and peroxisome proliferator-activated receptor $y$ coactivator 1-a (PGC-1a) genes in M. pectoralis $(P e c)$ and gastrocnemius (Gas) muscles of thermoneutral (TN) and heat-stressed (HS) chickens

\begin{tabular}{|c|c|c|c|c|}
\hline \multirow{2}{*}{ Genes } & \multicolumn{2}{|l|}{ Pec muscle } & \multicolumn{2}{|l|}{ Gas muscle } \\
\hline & $\mathrm{TN}$ & HS & $\mathrm{TN}$ & $\mathrm{HS}$ \\
\hline avUCP & $1.00 \pm 0.08$ & $0.64 \pm 0.05^{*}$ & $1.21 \pm 0.08$ & $1.18 \pm 0.05$ \\
\hline SOD2 & $1.00 \pm 0.11$ & $1.09 \pm 0.15$ & $1.53 \pm 0.11$ & $1.89 \pm 0.10^{*}$ \\
\hline SOD1 & $1.00 \pm 0.09$ & $1.12 \pm 0.08$ & $1.27 \pm 0.09$ & $1.10 \pm 0.08$ \\
\hline CAT & $1.00 \pm 0.09$ & $0.94 \pm 0.10$ & $0.96 \pm 0.09$ & $1.08 \pm 0.10$ \\
\hline GPx4 & $1.00 \pm 0.13$ & $1.11 \pm 0.06$ & $1.25 \pm 0.13$ & $1.29 \pm 0.06$ \\
\hline PGC-1a & $1.00 \pm 0.12$ & $0.94 \pm 0.07$ & $1.41 \pm 0.12$ & $1.60 \pm 0.07$ \\
\hline
\end{tabular}

Real-Time reverse transcription polymerase chain reaction (RT-PCR) was used to quantify the mRNA levels. The results were normalized to 18s-ribosomal protein (RN18S) transcript levels. Values are means \pm error, $n=6$, and data were expressed as relative values to $P e c$ muscle of TN birds; * indicates the difference between TN and HS chickens for each muscle separately at $P<0.05$

sibly a factor. However, it was found that the increase in muscle temperature following HS treatment did not differ between the muscles $\left(\Delta 2.4 \pm 0.4{ }^{\circ} \mathrm{C}\right.$ in $\mathrm{Pec}$ muscle; $\Delta 1.7 \pm 0.5^{\circ} \mathrm{C}$ in Gas muscle). Also, we further compared the degrees of HS-induced oxidative damage $(\%$ increase in TBARS values of HS condition compared to TN condition) in Pec, Gas and extensor digitorum longus (type IIB: 87\%) (Williams and Dhoot, 1992). HS-induced lipid peroxidation was shown to decrease as the percentage of glycolytic muscle fibre in the muscles decreased (data not shown), suggesting that oxidative status of muscles with a higher proportion of glycolytic muscle fibre might be more susceptible to HS.
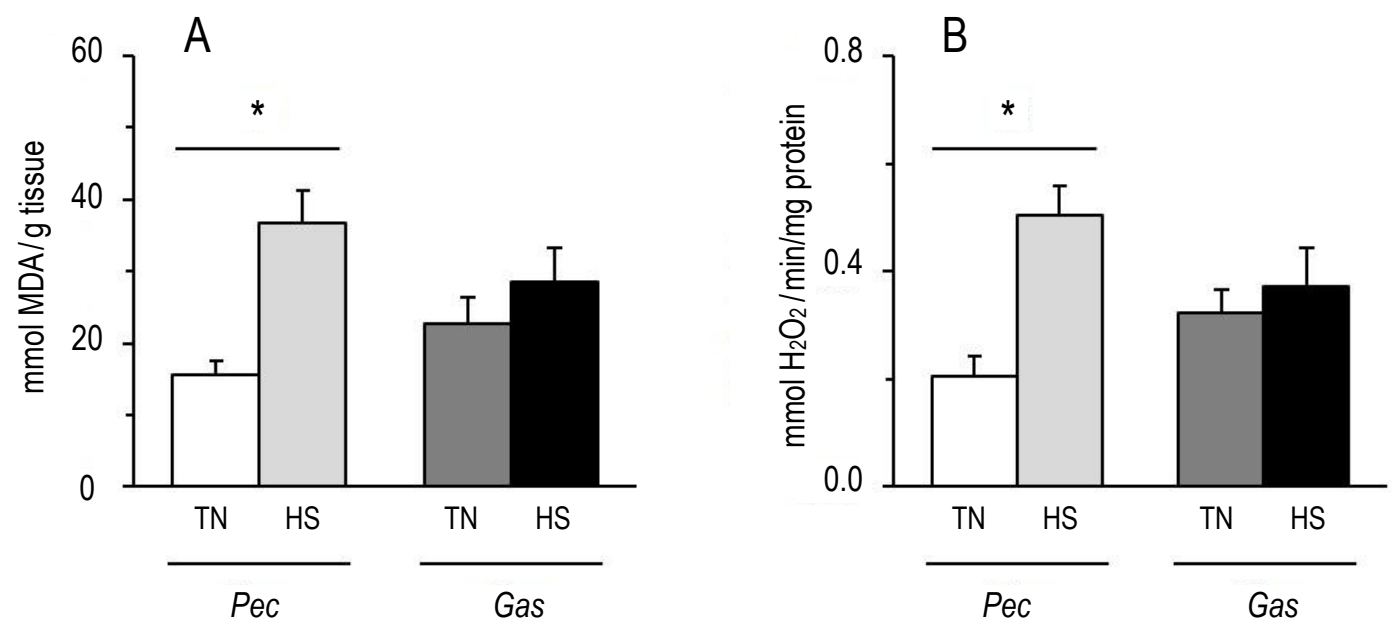

Figure 1. Lipid peroxidation (A) and mitochondrial $\mathrm{H}_{2} \mathrm{O}_{2}$ production $(\mathrm{B})$ in $\mathrm{Pec}$ (M. pectoralis) and Gas (gastrocnemius) muscles in thermoneutral (TN) and heat-stressed (HS) broiler chickens

Lipid peroxidation was measured using thiobarbituric acid (TBA), and is expressed as nmol of malondialdehyde (MDA) per equivalent $\mathrm{g}$ wet tissue. $\mathrm{H}_{2} \mathrm{O}_{2}$ production rate in isolated mitochondria oxidizing succinate $(4 \mathrm{mM})$ was fluorometrically determined using Amplex ${ }^{\top \mathrm{TM}}$ Red (Invitrogen, Carlsbad, CA, USA). Values are means \pm standard error, $n=6$; * indicates the difference between TN and HS chickens for each muscle separately at $P<0.05$ 
Apart from oxidative damage, in the present study it was shown that HS resulted in a significant increase in mitochondrial $\mathrm{H}_{2} \mathrm{O}_{2}$ production in $\mathrm{Pec}$ muscle, while in Gas muscle the increase was not observed (Figure 1B). Also, mechanism regulating mitochondrial ROS generation in these muscles under HS conditions was examined. It was found that down-regulation of avUCP, a mitochondrial inner membrane protein, evokes ROS generation through an increase in the inner membrane potential under hyperthermic conditions (Kikusato and Toyomizu, 2013).

It was shown that the avUCP mRNA level in $\mathrm{Pec}$ muscle was significantly decreased by the HS treatment, and did not change in Gas muscle (Table 1). Following the HS treatment, mRNA levels of SOD2, which is an antioxidative enzyme localized in the mitochondrial matrix, were unchanged in $\mathrm{Pec}$ muscle, while the levels of SOD2 in Gas muscle were significantly higher in HS birds than in TN birds. In addition, the study investigated the change in transcription rates of the cytosolic antioxidative enzymes: SOD1, CAT and GPx4; however, no significant differences in the mRNA levels were observed between TN and HS groups in both Pec and Gas muscles. These results suggest that maintained levels of avUCP and upregulation of SOD2 may play an important role in the suppression of mitochondrial ROS generation and subsequent oxidative disturbance in skeletal muscle of HS-exposed broiler chickens.

Skeletal muscle fibre type transformation between fast and slow fibre types is caused by physiological stimuli in order to fulfil metabolic demands. The present study did not evaluate these changes in $P e c$ and Gas muscles of HS-exposed birds; however, in a previous study it was reported that mild HS induces differentiation of mammalian myoblasts, shifting fibre type from glycolytic to oxidative through PGC-1 $\alpha$ (Yamaguchi et al., 2010). PGC-1 $\alpha$, a transcriptional co-factor, plays a pivotal role in the muscle fibre type shift (Zhang et al., 2017); however, the present study found that PGC- $1 \alpha$ mRNA levels were not altered by HS treatment in $P e c$ and Gas muscles (Table 1). Therefore, it is unlikely that HS induced muscle fibre transformation in the present study. Given that PGC- $1 \alpha$ induces the transcription of mitochondrial antioxidative factors (Valle et al., 2005), the mechanism maintaining avUCP mRNA level and up-regulating SOD2 in Gas muscle remains unclear. One might assume that other signalling molecules such as AMP-activated protein kinase (AMPK) and sirtuin-1, which act in coordi- nation with PGC-1 $\alpha$ in energy metabolism (Cantó and Auwerx, 2009), or the higher intrinsic levels of PGC-1 $\alpha$ mRNA (Gas muscle vs Pec, $P<0.05$ ), might be associated with the transcription of the mitochondrial antioxidative factors in Gas muscle of HS-exposed birds. Further investigation is required to clarify if specific muscles have a higher tolerance to oxidative disturbance caused by HS treatment.

The present study revealed that the oxidative status of Gas muscles did not respond to acute HS treatment. However, further investigations are needed to verify whether this finding is applicable to other animals. In a study on pigs it was reported that oxidative muscle is more susceptible to HS-induced changes in redox balance than glycolytic muscle during chronic HS (Rosado Montilla et al., 2014). Conversely, it was reported that HS-induced oxidative disturbance did not differ considerably between muscles with different fibre types: Gas, soleus, and plantaris muscles, in aged mice (Tamura et al., 2017). It is conceivable that the difference in HS intensity and duration between the studies might be associated with the discrepancies between these results, while one might assume that the muscle lipid content of the different animals used affected the degrees of HS-exposed oxidative damage.

\section{Conclusions}

It is suggested that skeletal muscles of broiler chickens that contain different amounts of glycolytic muscle fibre exhibit different responses to heat stress (HS)-induced oxidative disturbance, in which the mitochondrial antioxidative factors such as avian uncoupling protein (avUCP) and superoxide dismutase 2 (SOD2) may be involved.

\section{Acknowledgement}

This work was supported by the Japan Society for the Promotion of Science (JSPS) (KAKENHI grant numbers 25850182, $16 \mathrm{H} 06205$ and 15H04582) and by JSPS Core-to-Core Advanced Research Networks Program, entitled 'Establishment of international agricultural immunology research-core for a quantum improvement in food safety.'

\section{References}

Cantó C., Auwerx J., 2009. PGC-1a, SIRT1 andAMPK, an energy sensing network that controls energy expenditure. Curr. Opin. Lipidol. 20, 98-105, https://doi.org/10.1097/MOL.0b013e328328d0a4 
Hakamata Y., Watanabe K., Amo T., Toyomizu M., Kikusato M., 2018. Characterization of mitochondrial content and respiratory capacities of broiler chicken skeletal muscles with different muscle fiber compositions. J. Poult. Sci. 55, 210-216, https:// doi.org/10.2141/jpsa.0170141

Kikusato M., Nanto F., Mukai K., Toyomizu M., 2016. Effects of trehalose supplementation on the growth performance and intestinal innate immunity of juvenile chicks. Br. Poult. Sci. 57, 375-380, https://doi.org/10.1080/00071668.2016.1166475

Kikusato M., Ramsey J.J., Amo T., Toyomizu M., 2010. Application of modular kinetic analysis to mitochondrial oxidative phosphorylation in skeletal muscle of birds exposed to acute heat stress. FEBS Lett. 584, 3143-3148, https://doi. org/10.1016/j.febslet.2010.05.057

Kikusato M., Toyomizu M., 2013. Crucial role of membrane potential in heat stress-induced overproduction of reactive oxygen species in avian skeletal muscle mitochondria. PLoS ONE 8, e64412, https://doi.org/10.1371/journal.pone.0064412

Kikusato M., Toyomizu M., 2015. Moderate dependence of reactive oxygen species production on membrane potential in avian muscle mitochondria oxidizing glycerol 3-phosphate. J. Physiol. Sci. 65, 555-559, https://doi.org/10.1007/s12576-015-0395-2

Kikusato M., Yoshida H., Furukawa K., Toyomizu M., 2015. Effect of heat stress-induced production of mitochondrial reactive oxygen species on NADPH oxidase and heme oxygenase-1 mRNA levels in avian muscle cells. J. Thermal. Biol. 52, 8-13, https:// doi.org/10.1016/j.jtherbio.2015.04.005

National Research Council (NRC), 1994. Nutrient Requirements of Poultry: $9^{\text {th }}$ Revised Edition. The National Academies Press. Washington, DC (USA), https://doi.org/10.17226/2114
Picard M., Hepple R.T., Burelle Y., 2012. Mitochondrial functional specialization in glycolytic and oxidative muscle fibres: tailoring the organelle for optimal function. Am. J. Physiol. Cell Physiol. 302, C629-C641, https://doi.org/10.1152/ajpcell.00368.2011

Rosado Montilla S.I., Johnson T.P., Pearce S.C., Gardan-Salmon D., Gabler N.K., Ross J.W., Rhoads R.P., Baumgard L.H., Lonergan S.M., Selsby J.T., 2014. Heat stress causes oxidative stress but not inflammatory signaling in porcine skeletal muscle. Temperature 1, 42-50, https://doi.org/10.4161/temp.28844

Tamura Y., Matsunaga Y., Kitaoka Y., Hatta H., 2017. Effects of heat stress treatment on age-dependent unfolded protein response in different types of skeletal muscle. J. Gerontol. Ser. A Biol. Sci. Med. Sci. 72, 299-308, https://doi.org/10.1093/gerona/glw063

Valle I., Álvarez-Barrientos A., Arza E., Lamas S., Monsalve M., 2005. PGC-1a regulates the mitochondrial antioxidant defense system in vascular endothelial cells. Cardiovasc. Res. 66, 562573, https://doi.org/10.1016/j.cardiores.2005.01.026

Williams K., Dhoot G.K., 1992. Heterogeneity and distribution of fast myosin heavy chains in some adult vertebrate skeletal muscles. Histochemistry 97, 361-370, https://doi.org/10.1007/ BF00270039

Yamaguchi T., Suzuki T., Arai H., Tanabe S., Atomi Y., 2010. Continuous mild heat stress induces differentiation of mammalian myoblasts, shifting fiber type from fast to slow. Am. J. Physiol. Cell Physiol. 298, C140-C148, https://doi.org/10.1152/ajpcell.00050.2009

Zhang L., Zhou Y., Wu W., Hou L., Chen H., Zuo B., Xiong Y., Yang J., 2017. Skeletal muscle-specific overexpression of PGC-1a induces fiber-type conversion through enhanced mitochondrial respiration and fatty acid oxidation in mice and pigs. Int. J. Biol. Sci. 13, 1152-1162, http://doi.org/10.7150/ijbs.20132 